\title{
Validation of Wireless Sensor Networks with Cross-Layer Properties
}

\author{
Vemula Harini \\ Assistant Professor \\ Vardhaman College of Engineering \\ Hyderabad, INDIA
}

\author{
Barupati Sampath \\ Student of M.Tech \\ Vardhaman College of Engineering \\ Hyderabad, INDIA
}

\begin{abstract}
In the original OSI networking model, data are kept strictly within a given layer because strict boundaries between layers are enforced. Cross layer optimization removes such strict boundaries to allow communication between layers by permitting one layer to access the data of another layer to exchange information and enable interaction. In this paper, the working is based on the comprehensive cross-layer analysis framework, which employs a stochastic queuing model in realistic channel environments. This framework is generic and can be parameterized for a wide variety of MAC protocols and routing protocols.
\end{abstract}

\section{Keywords}

OSI, QOS, WSN, ROUTING PROTOCOLS.

\section{INTRODUCTION}

Emerging applications of wireless sensor networks require real-time quality-of-service guarantees to be provided by the network. Because of the nondeterministic impacts of the queuing mechanisms and wireless channel, probabilistic analysis of QoS is essential. A wireless sensor network (WSN) consists of sensor nodes capable of collecting information from the environment and communicating with each other via wireless transceivers. With multi-hop communication the collected data will be delivered to one or more destinations. The sensor nodes are characteristic's to identify expected to operate with batteries and are often deployed to not-easily-accessible or hostile environment, sometimes in large quantities. To find the optimal communication route from source to destination is only basic and main goal of routing in WSN. Optimal path considers the other network factors as well such as latency, jitter, network originating from above, throughput, communication cost and power in order to communicate between the source and destination without failure.

Due to mobility the communication paths are changing very frequently and hence network packets are not at all affected or even not changing the packet optimality and its uniformity.

There are mainly three categories of the mobile routing protocols such as proactive, reactive and hybrid routing protocols as shown following Fig 1 . There are many protocols which here considering for the investigation and evaluation in the mobile ad hoc networks. But each of these routing protocols is focused on the certain aspects of simulation results TCP is not well suited for wireless networks especially in WSN; the performance of TCP degrades significantly due to the heavy .

To overcome the problems of reliability, versions of TCP called TCP variants were developed especially for wireless ad hoc networks to provide reliable communication. There are different network layer protocols for route discovery and maintenance in WSN but, the issue is the selection of suitable coupling of TCP variant over WSN routing protocol to provide reliable communication.

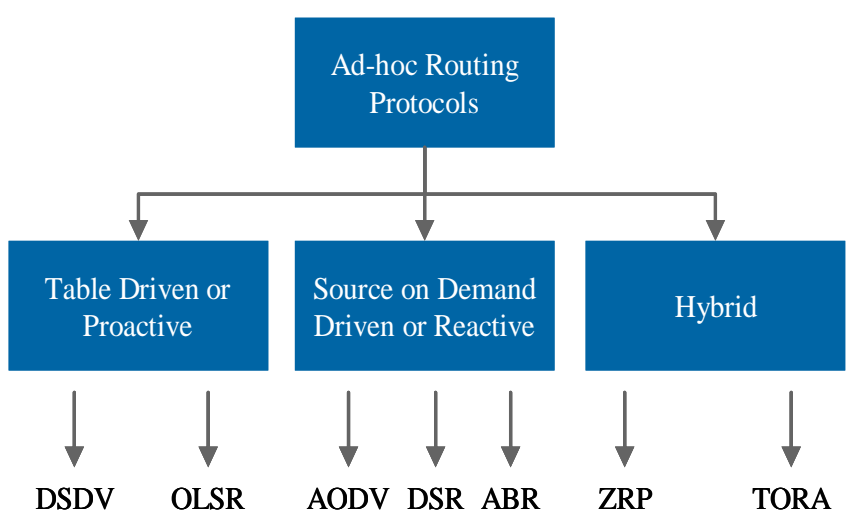

Fig1. Routing Protocols

\section{STUDY OF DSDV AND AODV DSR ROUTING PROTOCOLS}

\subsection{Destination Sequenced Distance Vector (DSDV)}

DSDV is one of the most well known table-driven routing algorithms. The DSDV routing algorithm is based on the number of hops to reach to the destination, sequence number of the classical Bellman-Ford Routing Algorithm with certain improvement. Each and every mobile node maintains a routing table with all available destinations along with some more information. The advantages of DSDV is an adaptation of classical distance vector routing protocol to ad hoc networks. It is quite suitable for creating ad hoc networks with small number of nodes.Now, disadvantages of DSDV needs regular update of its routing tables, which wastes battery power and a small amount of bandwidth even when the network is idle.

If the topology of the network changes, a new sequence number is necessary before the network re-converges; thus, DSDV is not suitable for highly dynamic networks.

\subsection{Ad-hoc On-demand Distance Vector (AODV)}

Reactive protocols discover routes when it's required. If a node wishes to communicate with another node, it checks with its previous information for a valid route to the destination. If one route is found, the node uses that route for communication with the destination node. If route is not found, the source node starts a route discovery process by RREQ, to which either the destination node or one of the intermediate nodes sends a reply back to the source node with a valid route. Less amount of information (mostly fixed packet size) is stored into routing packet unlike DSR routing 
protocol. The Advantages of AODV route discovery process is in on demand, which is more efficient in dynamic nature of mobile ad-hoc network .Disadvantages of AODV is Due to on demand manner, it won't check route in periodic interval so transmission of data after discover the rote is taking some delay, but due to dynamic nature of network this delay is not considerable.

\section{RELATED WORK}

In paper [1], defines a quantitative notion of real-time capacity of a wireless network. This describes how much realtime data the network can transfer by their deadlines. For using as a sufficient schedulability condition for a class of fixed-priority packet scheduling algorithms a capacity bound is derived that can be used.

\subsection{Pros and Cons of real time data network}

This paper presents the first expressions for real-time capacity of a sensor network and derived a sufficient condition for schedulability under fixed-priority scheduling which allows capacity planning to be employed prior to deployment such that real-time requirements are met at runtime but it does not considers about variable density networks, realistic MAC-layers, and effects of energy constraints to name a few.

In paper [2], author surveys recent routing protocols for sensor networks and presents a classification for the various approaches pursued. Author mainly explored three main categories in this paper those are hierarchical, data-centric and location-based. All routing protocols are described and discussed under the appropriate category and all protocols are using contemporary methodologies such as network flow and QoS modeling, which are also discussed.

\subsection{Pros and Cons of routing protocols}

The author was summarized recent research results on data routing in sensor networks and classified the approaches into three main categories, namely hierarchical, data-centric and location-based. Few other protocols followed the traditional network flow and QoS modeling methodology. However, there are some hybrid protocols that fit under more than one category but further research would be needed to address issues such as Quality of Service (QoS) posed by video and imaging sensors and real-time applications.

In paper [3], author discussed about the state of the art in protocols, algorithms and hardware for wireless multimedia sensor networks is surveyed and open research issues are discussed in detail. WMSN architectures are explored along with their advantages and drawbacks. Currently available research prototypes as well as the off-the-shelf hardware for WMSNs are listed and classified.

\subsection{The Pros and Cons of WMSN}

Author discussed the state of the art of research on Wireless Multimedia Sensor Networks (WMSNs), and outlined the main research challenges. Algorithms, protocols, and hardware for the development of WMSNs were surveyed, and open research issues discussed in detail.

In paper [4], author described the concept of sensor networks which has been made viable by the convergence of wireless communications micro-electro mechanical system and digital electronics First, the potential sensor networks and the sensing tasks applications are explored and a review of factors influencing the design of sensor networks is provided and then the communication architecture for sensor networks is outlined and the algorithms and protocols developed for each layer in the literature are explored.

Pros and Cons of different parameters:

The fault tolerance, flexibility, low-cost, high sensing fidelity and rapid deployment characteristics of sensor networks create many new and exciting application areas for remote sensing. Further this wide range of application areas will make sensor networks an integral part of our lives but these constraints are highly stringent and specific for sensor networks.

In paper [5], author have focused on characterizing the average end-to-end delay and maximum achievable per-node throughput in random access multi-hop wireless ad hoc networks with stationary nodes. Author presented an analytical model that takes into account the random packet arrival process, the number of nodes, the extent of locality of traffic and the back off and collision avoidance mechanisms of random access MAC.

\subsection{Pros and Cons of Analytical Model}

In this delay analysis of random access MAC multi-hop wireless ad hoc networks. Here showed the closed form of expressions for the average end-to-end delay and maximum achievable throughput for comparable network parameters and the upper bound on maximum achievable throughput is of the same order as the Gupta-Kumar's bound.

\subsection{Existing system}

This is the first work of transport to physical layer that provides a generic, probabilistic cross-layer analysis of endto-end delay in WSNs.

Previous method employs discrete-time queuing theory to analyze the end-to- end delay of wireless multi-hop networks for two MAC schemes, m-phase TDMA and slotted ALOHA (not cross layer analyze).

\subsection{Disadvantages}

Existing system can be observed that completely and accurately characterizing end-to-end delay in WSNs is still an open problem

Existing analysis did not capture a spacious change of MAC protocols with duty cycle operation and a case study with any cast protocol is included.

\subsection{Proposed system}

Here extend previous work, model is extended to capture a wide variety of MAC protocols with duty-cycle operation and a case study with an any cast protocol is included. Moreover, considering the various deployment methodologies in WSN applications, such as both deterministic and random network topologies are analyzed.

\subsection{Advantage of proposed system}

The proposed frame work can exact models the distribution of the end to end delay and captures the heterogeneous effects of multi hop WSNs. For the development of QoS based scheduling and communication solutions for WSNs the proposed framework can be used.

\section{TCP}

\subsection{RENO}

The Reno TCP implementation retained the enhancements to Tahoe, but changed the Fast Retransmit operation to include 
Fast Recovery [Jac90]. This algorithm prevents the communication path from going empty after Fast Retransmit, because of that avoiding the need to Slow-Start to re-fill it after a single packet loss.

In Reno, the sender's usable window becomes min(awin, cwnd+ndup) where awin is the receiver 's advertised window, cwnd is the sender' $\mathrm{s}$ congestion window, and ndup is maintained at 0 until the number of dup ACKs reaches tcp thresh, hence forth tracks the number of duplicate ACKs. Thus, during Fast Recovery the sender "inflates" its window by the number of dup ACKs it has received, accordant with the observation that each dup ACK indicates some packet has been removed from the network and is now cached at the receiver.

\subsection{TCP VEGAS}

TCP Vegas was proposed by Brakmo et al. It has a very different congestion control algorithm compared to New Tahoe. TCP Vegas in general controls its segment flow rate based on its estimate of the available network bandwidth. Among the many new features implemented in TCP Vegas, the most important difference between it and TCP Tahoe lies in its bandwidth estimation scheme. Studies on TCP Vegas have shown that Vegas achieve higher efficiency than Tahoe, causes fewer packet re transmissions.

Slow-start

W-init $\leftarrow 1$;

delta $\leftarrow(\mathrm{W} / \mathrm{RTTmin}-\mathrm{W} / \mathrm{RTT}) * \mathrm{RTTmin} ;$

For each ack

If (delta<gamma)then

$\mathrm{W} \leftarrow \mathrm{W}+1 ;$

else

enter Congestion Avoidance

end if

Congestion Avoidance

delta $\leftarrow($ W/RTTmin-W/RTT)*RTTmin;

for each ack

if (delta<alpha)then

$\mathrm{W} \leftarrow \mathrm{W}+1 / \mathrm{W} ;$

else if (delta>beta)then

$\mathrm{W} \leftarrow \mathrm{W}-1 ;($ only execute once in a RTT)

else

$\mathrm{W} \leftarrow \mathrm{W} ;$

end if

Fast Retransmit

if(dup_ACKS)

retransmit the lost packet

$\mathrm{W} \leftarrow \mathrm{W} / 2 ;$

enter Congestion Avoidance

\subsection{New Reno}

TCP New Reno defined by RFC 3782, advances retransmission during the fast recovery phase of TCP Reno. while fast recovery for every duplicate ACK that is returned to TCP New Reno, a new not sent packet from the end of the congestion window is sent, to keep the transmit window as full.

For each and every ACK that provides related to progress in the sequence space, sender assumes that the ACK points to a new hole and the next packet beyond the ACK sequence number is sent.

In this paper analysed the different layer property by the quality of service parameters like packet delivery ratio, End to End delay, and overhead and convergence time.

\subsection{Packet delivery function}

Packet delivery function is the defined as number of packets successfully transmitted b/w source and destination.

\subsection{End to End delay}

Time duration $\mathrm{b} / \mathrm{w}$ packet received and sending time is called as End to End delay.

\subsection{Overhead and Convergence time}

Number of extra packets such as routing packets called as overhead. The convergence time is defined as time duration between route failure and route recovery

\section{PERFORMANCE EVALUATION}

In this paper (cross layer analysis) with extension, to determine the packet delivery function using number of routing protocols (such as AODV, DSDV, DSR) and also CBR traffic and various TCP protocols and Physical layer changes. In this paper with extension, with more parameters like packet delivery, overhead and also delay etc,

For this paper execution studied the different network environment by using NS2 tool. In this paper, here showing in this various network environments given in below table.

Here, comparing the different routing protocols along with TCP protocol in different network environment. Simulation result for packet delivery ratio is shown in the following graphs ; TCP New Reno is mostly providing high performance with DSR and AODV but not in DSDV.

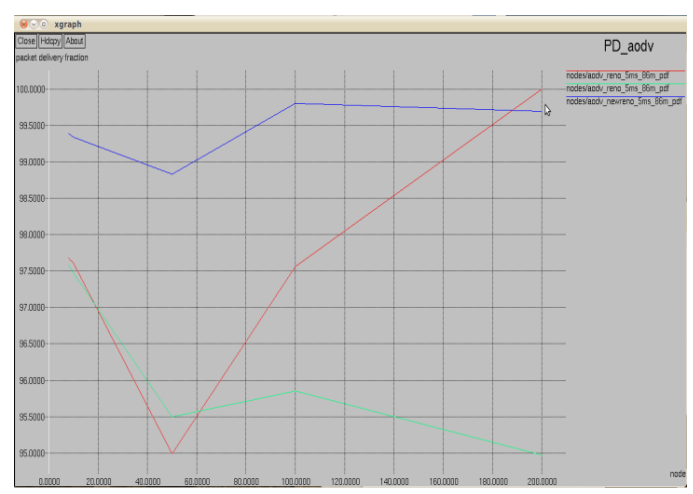

Fig.2 Packet delivery function with AODV (varying nodes)

end if 


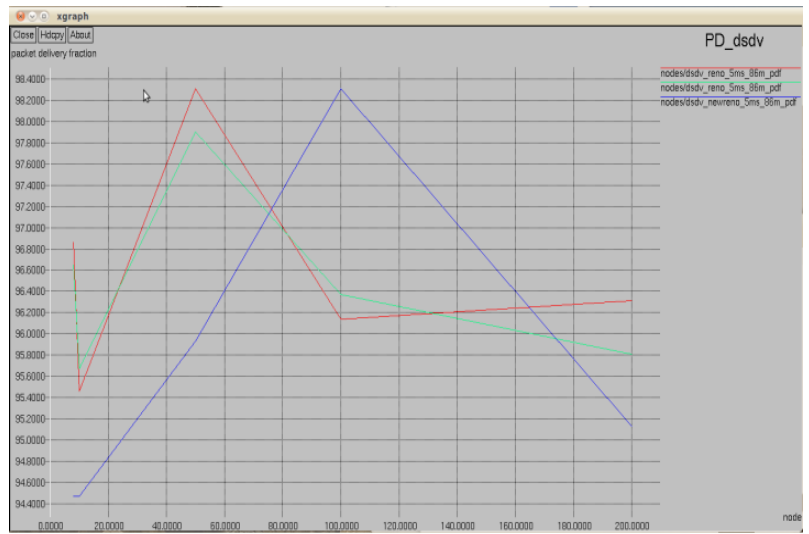

Fig3. Packet delivery with DSDV (varying nodes)

In next comparison, the measured variable as coverage area. In this result also TCP New RENO is better than remaining TCP protocols

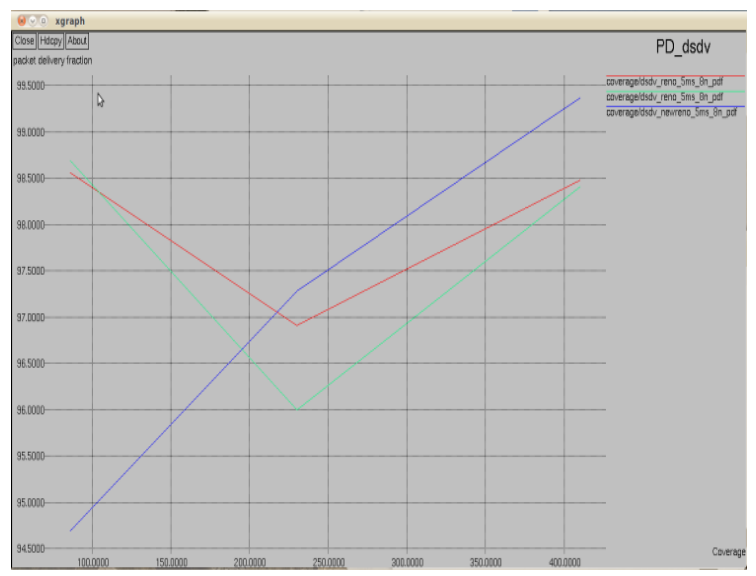

Fig 4.Packet delivery function wit AODV(coverage)

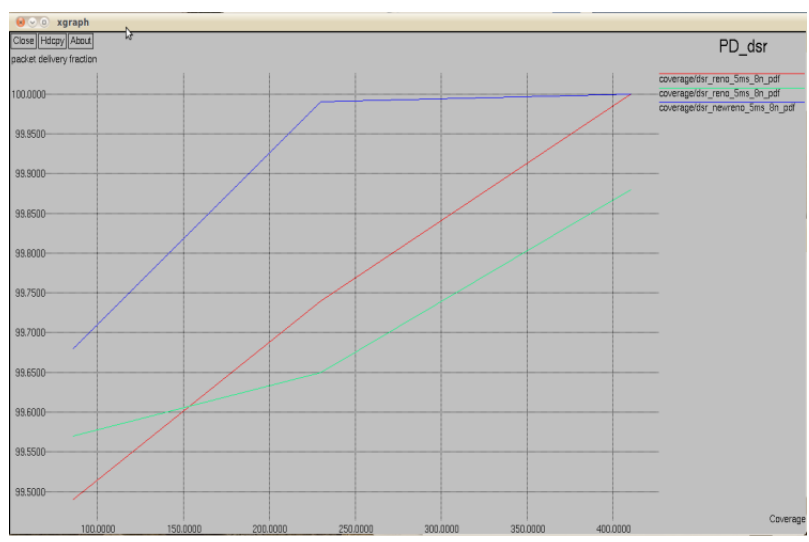

Fig 5. Packet delivery with DSR(coverage)

In End to End delay analysis, Reno providing constant delay in different network environment in all the routing protocol. But new Reno is proving less delay in 100 node environment for all routing protocols.

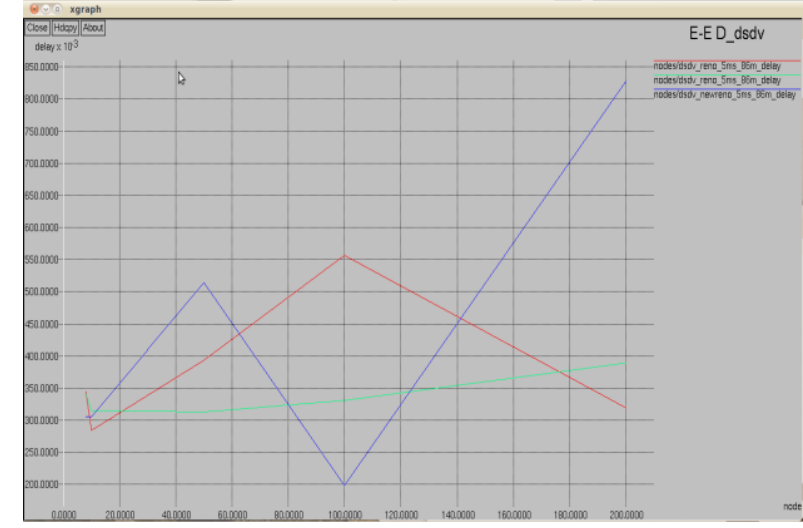

Fig 6.End to End delay with DSDV(nodes)

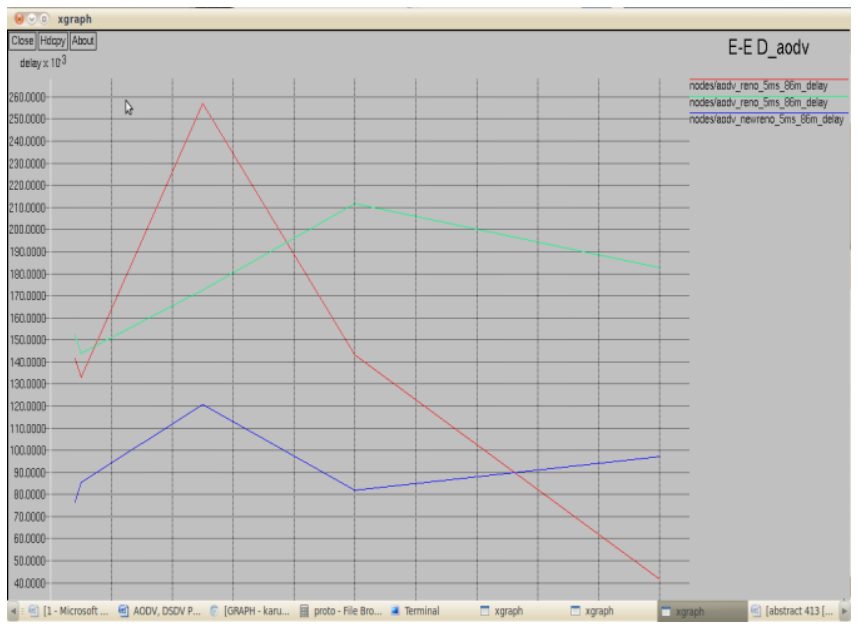

Fig 7. End to End Delay with AODV

\begin{tabular}{|l|l|}
\hline Parameter name & Parameter value \\
\hline Area & $\begin{array}{l}500 \times 500,700 \times \text { X 700, } \\
1600 \times 1600\end{array}$ \\
\hline Coverage area & $86 \mathrm{~m}, 230 \mathrm{~m}, 410 \mathrm{~m}$ \\
\hline Routing protocol & AODV, DSR, DSDV \\
\hline Transport protocol & $\begin{array}{l}\text { TCP--- Reno, Vegas ,New } \\
\text { Reno }\end{array}$ \\
\hline No. Of nodes & $8,10,50,100,200$ \\
\hline Speed & $5 \mathrm{~m} / \mathrm{s}, 10 \mathrm{~m} / \mathrm{s}, 20 \mathrm{~m} / \mathrm{s}$ \\
\hline MAC & $\mathrm{MAC} / 802.11$ \\
\hline &
\end{tabular}

In convergence time analysis, DSR providing less convergence time in most of the cases. 


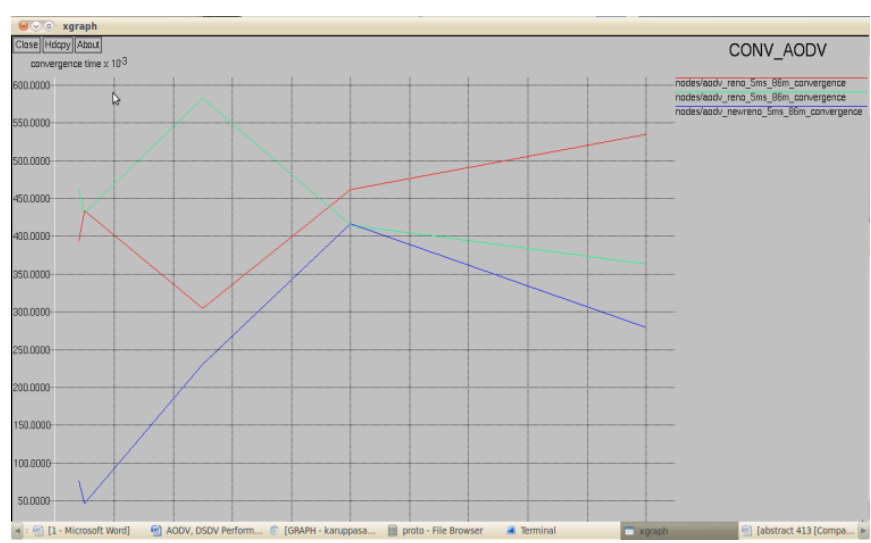

Fig 8. Convergence time with AODV(varying nodes)

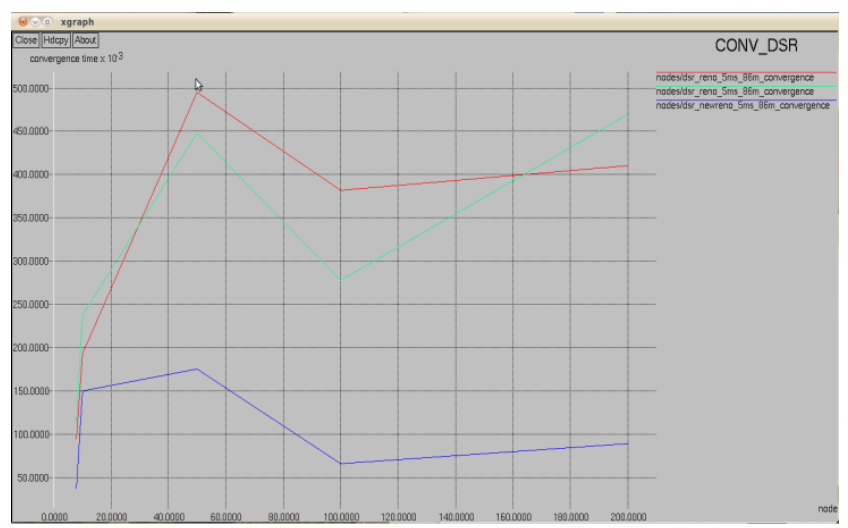

Fig 9. Convergence time with DSR (varying nodes)

\section{CONCLUSION}

In this paper, an end-to-end analysis of the communication delay and packet delivery function and convergence time is provided. The developed framework using ns2 simulator can be used to guide the development of QoS-based scheduling and communication solutions for WSNs.

\section{ACKNOWLEDGMENTS}

The authors would like to thank the Vardhaman college of engineering for its support with their excellent lab facilities.

\section{REFERENCES}

[1] Tarek F. Abdelzaher, Shashi Prabh, Raghu Kiran, "On Real-time Capacity Limits of Multi hop Wireless Sensor Networks" - 2004.

[2] Kemal Akkaya and Mohamed ,"A Survey on Routing Protocols for Wireless Sensor Networks" - 2005.

[3] Ian F. Akyildiz, Tommaso Melodia, Kaushik R. Chowdhury, "A survey on wireless multimedia sensor networks" - 2007.

[4] I.F. Akyildiz, W. Su, Y. Sankarasubramaniam, E. Cayirci "Wireless sensor networks: a survey" - 2002.

[5] Nabhendra Bisnik, Alhussein Abouzeid, "Queuing Network Models for Delay Analysis of Multi hop Wireless Ad Hoc Networks" - 2006.

[6] Almut Burchard, JorgLiebeherr, Stephen Patek "A MinPlus Calculus for End-to-end Statistical Service Guarantees" - 2006.

[7] Emad Felemban, Chang-Gun Lee, Eylem Ekici, Ryan Boder and Serdar Vural, "Probabilistic QoS Guarantee in Reliability and Timeliness Domains in Wireless Sensor Networks" - 2005

[8] Gagan Raj Gupta and Ness Shroff "Delay Analysis for Multi-hop Wireless Networks" - 2009.

[9] John P. Lehoczky, "Real-Time Queuing Theory" - 1996.

[10] Abusayeed Saifullah, You Xu, Chenyang Lu, andYixin Chen, "End-to-End Delay Analysis for Fixed Priority Scheduling in Wireless HART Networks"

[11] Oliver Hahm, Emmanuel Baccelli, Mesut Günes, Matthias Wählisch, Thomas C. Schmidt, RIOT OS: Towards an OS for the Internet of Things, In: Proc. of the 32nd IEEE INFOCOM. Poster Session, Piscataway, NJ, USA:IEEE Press, 2013.

[12] J.D. Kenney, D.R. Poole, G.C. Willden, B.A. Abbott, A.P. Morris, R.N. McGinnis, and D.A. Ferrill, "Precise Positioning with Wireless Sensor Nodes: Monitoring Natural Hazards in All Terrains",2009 IEEE International Conference on Systems, Man, and Cybernetics, San Antonio, TX, USA, Oct. 2009. 